\title{
Alginat bei Bedarf als Add-on bei unzureichendem Effekt von Protonenpumpen-Hemmern bei Patienten mit gastroösophagealer Reflux-Krankheit
}

\section{Alginate on demand as add-on for patients with gastro-oesophageal reflux disease and insufficient $P P I$ effect}

Autoren

Matthias Müller ${ }^{1}$, Gisela Labenz ${ }^{2}$, Daniela-P. Borkenstein ${ }^{1}$, Joachim Labenz ${ }^{1}$, LOPA Studiengruppe

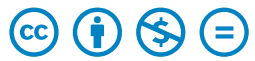

Institute

1 Diakonie-Klinikum, Jung-Stilling-Krankenhaus, Siegen

2 Reflux-Zentrum Siegerland, Medizinisches Zentrum Siegerland Flughafen, Burbach

Schlüsselwörter

Alginat, gastroösophageale Reflux-Krankheit (GERD), PPI-refraktäre Symptome, Säurekontrolle, Reflux-Kontrolle

Key words

acid control, alginate, gastro-oesophageal reflux disease (GORD), PPI refractory symptoms, reflux control

Bibliografie

DOI https://doi.org/10.1055/a-0736-5959

Online-Publikation: 22.11.2018

Dtsch Med Wochenschr 2019; 144: e30-e35

(c) Georg Thieme Verlag KG, Stuttgart · New York

ISSN 0012-0472

\section{Korrespondenzadresse}

Prof. Dr. med. Joachim Labenz

Direktor Innere Medizin

Diakonie Klinikum, Jung-Stilling-Krankenhaus,

Wichernstr. 40, 57074 Siegen

J.Labenz@t-online.de

\section{ZUSAMMENFASSUNG}

Einleitung Chronische Reflux-Patienten mit Langzeit-PPITherapie haben häufig persistierende Symptome. Eine wesentliche Ursache hierfür ist die Acid-Pocket, die lokal durch Alginate wirksam behandelt werden kann.

Methoden In einer prospektiven, multizentrischen, nicht randomisierten, nicht interventionellen und unkontrollierten Studie in hausärztlichen Praxen wurden Reflux-Patienten mit einer PPI-Dauertherapie über $\geq 1 \mathrm{Jahr}$, die mit ihrer Behandlung unzufrieden waren, über 14 Tage mit einem Alginat nach Bedarf (bis zu $4 \times / T a g$ ) behandelt. Es wurde die Frequenz der Symptome in der Woche vor Therapie und in der zweiten Behandlungswoche erfasst, zusätzlich der validierte GERD-Q-Score. Die Patienten gaben ihre Zufriedenheit mit der Therapie vor Beginn und am Ende der Studienperiode auf einer 5-Punkt-Likert-Skala an (1: sehr unzufrieden; 5: zufrieden). Primärer Endpunkt der Studie war die Verbesserung der Zufriedenheit um $\geq 1$ Punkt. Ergebnisse 155 Patienten (54\% Frauen, medianes Alter 57 Jahre [Bereich 18 - 85 Jahre]) wurden rekrutiert. 6 Patienten wurden von der Wirksamkeitsanalyse ausgeschlossen, da sie entweder die Studienmedikation nicht genommen hatten oder nicht zur Abschlussvisite erschienen. Die mediane Krankheitsdauer lag bei 8 Jahren (Bereich 1 - 50 Jahre), die mediane Dauer der PPI-Langzeittherapie bei 6 Jahren. In der Studie nahmen die Patienten im Mittel 3 Sachets des Studienmedikaments pro Tag. Die Alginat-Therapie führte bei $72 \%$ der Patienten zu einer Verbesserung der Therapiezufriedenheit um mindestens 1 Punkt (1 Punkt: $n=45,2$ Punkte: $n=41$, 3 Punkte: $n=16,4$ Punkte: $n=5$ ). Patienten mit einem GERDQ-Score $>8$ sprachen häufiger auf die Therapie an (81\%). Die Verbesserung umfasste alle typischen Reflux-Symptome und Schlafstörungen. Die Therapie wurde gut vertragen.

Diskussion Eine Reflux-Blockade mit einem Alginat als Addon ist eine wirksame und sichere Option zur Behandlung von Durchbruchsymptomen bei GERD-Patienten mit PPI-Therapie.

\section{ABSTRACT}

Background Chronic reflux patients with long-term PPI therapy frequently have persistent symptoms. A critical source of the persistent acid reflux is the acid pocket, which can be treated effectively by the non-systemic reflux suppressing action of alginates.

Methods GERD patients with at least one year of PPI longterm therapy, who were dissatisfied with their therapy, were treated over a period of 14 days with alginate as add-on and when required (up to 4 times daily) in a prospective, multicentre, non-randomised, non-interventional and uncontrolled study in GP practices. The frequency of symptoms in the week before treatment was recorded, as well as in the second week of the study phase and the consumption of alginate. The validated GERD Q score was also applied. The patients assessed satisfaction with the therapy on a five-point Likert scale (very unhappy - happy) before the beginning and at the end of the study. The primary endpoint of the study was improvement in satisfaction with the therapy by $\geq 1$ point. 
Results 155 patients (54\% women, median age $57(18-85)$ years) entered the study. Six patients had to be excluded from the final efficacy analysis (no intake of study drug, lost to follow-up). The median duration of GORD was $8(1-50)$ years and the median time on PPI therapy was 6 years. On average the patients took 3 sachets of alginate per day. The alginate therapy led to an improvement by at least 1 point on the Likert scale in $72 \%$ of patients ( 1 step $n=45,2$ steps $n=41$,
3 steps $n=16,4$ steps $n=5$ ). Patients with a Gerd Q score $>8$ showed a higher response rate at $81 \%$. There was improvement in all typical reflux symptoms and sleep disorders. The treatment was generally well-tolerated.

Conclusions A reflux blocking alginate taken on demand is an effective and safe option for the treatment of breakthrough symptoms in GERD patients on PPI.

\section{Einleitung}

Die gastroösophageale Reflux-Krankheit (GERD) ist häufig. Es wird geschätzt, dass jeder 4. bis 5. Erwachsene an einer GERD leidet [1]. Protonenpumpenhemmer (PPI) gelten als die Medikamente der ersten Wahl [2]. Sie haben die Behandlung der GERD revolutioniert, da bis zu ihrer Einführung nur wenig wirksame Therapieoptionen zur Verfügung standen.

PPI können aber nicht als ideale Medikation für diese Erkrankung gelten, da sie in die Pathophysiologie - Inkompetenz der Anti-Reflux-Barriere - nicht eingreifen. Sie heben den pH-Wert des Mageninhalts und damit des Refluats an. Damit werden Symptome und Läsionen, die durch Säureexposition des Ösophagus entstehen, günstig beeinflusst. Überzeugende Belege für die Wirksamkeit wurden insbesondere bei der Heilung erosiver Ösophagitiden aufgezeigt [3]. In nahezu allen Studien und entsprechenden Metaanalysen war der Effekt auf die Symptomkontrolle deutlich schlechter. Etwa 30 - 50 \% der Patienten bleiben symptomatisch [4]. Seltene und leichte Symptome werden zumeist noch akzeptiert; Symptome, die $\geq 2 \times$ pro Woche auftreten oder von moderater bis schwerer Qualität sind, schränken dagegen die Lebensqualität und damit die Akzeptanz ein. In einer vorangegangenen Studie in hausärztlichen Praxen konnten wir zeigen, dass nahezu jeder zweite chronische GERD-Patient mit PPI-Dauertherapie an mindestens 2 Tagen pro Woche Symptome hat und jeder 5. mit der Behandlung unzufrieden ist [5].

Forschungen der letzten Jahre haben gezeigt, dass die AcidPocket, eine physiologische, unmittelbar postprandial auftretende Säureansammlung direkt unterhalb der Cardia, bei Patienten mit defekter Anti-Reflux-Barriere Ursache für insbesondere postprandial auftretende Reflux-Symptome sein kann [2, 6]. Diese AcidPocket lässt sich durch eine konventionelle PPI-Therapie nicht eliminieren. Alginate fallen bei Säurekontakt aus und bilden eine gallertartige Schicht auf dem Mageninhalt. Hierdurch wird die Acid-Pocket eliminiert und Reflux-Episoden reduziert [7, 8]. Nach Kontakt mit dem Ösophagus-Epithel entsteht ein über mehr als 60 Minuten anhaftender Alginat-Film [9].

Da Alginate unmittelbar nach der Einnahme wirken, formulierten wir die Hypothese, dass mit einer derartigen Therapie als Add-on bei klinischem Bedarf die Patientenzufriedenheit mit ihrer GERD-Behandlung verbessert werden kann.

\section{Patienten und Methoden}

In einer offenen, multizentrischen, nicht randomisierten, nicht interventionellen und unkontrollierten Studie wurde die Wirksamkeit und Verträglichkeit einer Bedarfstherapie mit einem AlginatAdd-on bei unzureichendem PPI-Effekt untersucht. Die Studie wurde in 11 hausärztlichen Praxen in Nordrhein-Westfalen und Rheinland-Pfalz sowie dem Reflux-Zentrum Siegerland zwischen März 2016 und Oktober 2017 durchgeführt. Die Studie wurde gemäß der aktuellen Version der Deklaration von Helsinki durchgeführt und von den zuständigen Ethikkommissionen begutachtet und befürwortet (Erstvotum Ethikkommission der Ärztekammer Westfalen-Lippe vom 15.01.2016, AZ 2015-747-f-S; Zweitvotum Ethikkommission der Ärztekammer Rheinland-Pfalz vom 25.10.2016, 837.430.16 (10 749)). Die Studie wurde am 23.05.2016 im deutschen Register Klinischer Studien unter der Nummer DRKS00 010473 registriert.

\section{Einschlusskriterien}

Aufgenommen wurden Patienten beiderlei Geschlechts $\geq 18$ Jahre mit einer mindestens 1 -jährigen Anamnese einer GERD sowie einer PPI-Dauertherapie von $\geq 1$ Jahr, die mit ihrer Behandlung im Hinblick auf die Symptomkontrolle nicht zufrieden waren.

\section{Ausschlusskriterien}

Ausgeschlossen wurden Patienten, die die Einschlusskriterien nicht erfüllten, in der Schwangerschaft oder Stillzeit, mit bekannter Unverträglichkeit gegen Alginate, die neben der PPI-Therapie ein anderes Antazidum oder ein anderes Medikament zur Therapie der Reflux-Krankheit einnahmen, Patienten mit anamnestischen Hinweisen auf eingeschränkte Nierenfunktion und Herzinsuffizienz, Hypercalcämie, Nephrokalzinose und wiederholt aufgetretenen kalziumhaltigen Nierensteinen sowie Patienten mit fehlendem Einverständnis.

\section{Studienziele}

Primäres Studienziel war die Verbesserung der Patientenzufriedenheit mit der Therapie um $\geq 1$ Punkt auf einer 5-stufigen Likert-Skala (sehr unzufrieden - zufrieden) am Ende der 2. Behandlungswoche. Sekundäre Studienziele umfassten die Verträglichkeit und Sicherheit der Alginat-Therapie sowie die Wirkung auf typische Reflux-Symptome und Schlafstörungen in Woche 2. 


\section{Studienspezifische Maßnahmen}

Im Rahmen der Eingangsvisite wurden neben demografischen und krankheitsspezifischen Daten die Häufigkeit der typischen Reflux-Symptome in der vorangegangenen Woche erfasst und der GERD-Q-Score ermittelt - ein validierter Score zur Diagnose einer GERD [10]. Patienten, die sich für die Studie qualifizierten und einer Teilnahme zustimmten, erhielten als Studienmedikation ein Alginat (Gaviscon dual ${ }^{\circledR}$, bestehend aus $500 \mathrm{mg}$ Natriumalginat, $213 \mathrm{mg}$ Natriumhydrogencarbonat, $325 \mathrm{mg}$ Calciumcarbonat). Es war erlaubt, bis zu $4 \times$ täglich 1 - 2 Sachets einzunehmen. Die Einnahme wurde mit der zugehörigen Tageszeit in einem Tagebuch protokolliert. Nach 14 Tagen erfolgte eine erneute Befragung mit Berechnung des GERD-Q-Scores und patientenseitiger Einschätzung der Therapiezufriedenheit. Zudem wurden unerwünschte Ereignisse erfasst. Die Patienten wurden nach Abschluss auch noch zu ihrer Präferenz der Therapieform befragt.

\section{Statistische Analysen}

Wenngleich eine formale Stichprobenumfangsbestimmung für Studien explorativen Charakters nicht erforderlich ist, wurden Fallzahlüberlegungen angestellt, um zu bestätigen, dass eine hinreichend präzise Schätzung des Parameters von primärem Interesse zu erwarten ist. Der Parameter von primärem Interesse ist der Anteil der Patienten mit einer Verbesserung der Behandlungszufriedenheit nach 14 Tagen um mindestens 1 Punkt auf einer 5-stufigen Likert-Skala ( 1 = sehr unzufrieden bis $5=$ sehr zufrieden).

Ausgehend von einer Fallzahl von ca. 150 Patienten (ca. 133 auswertbare Patienten unter Berücksichtigung einer Drop-OutRate im Bereich von 10-15\%) wird geschätzt, dass die Spanne des $95 \%$-Konfidenzintervall für den Anteil der Patienten mit einer Verbesserung der Behandlungszufriedenheit maximal $\pm 8,5$ Prozentpunkte um den Punktschätzer umfasst.

Die Darstellung der Daten erfolgte deskriptiv mit Angabe von absoluten und relativen Häufigkeiten, Mittelwert $( \pm S D)$ bzw. Median (Spanne bzw. interquartiler Bereich). Die Analyse erfolgte auf der Basis der tatsächlich erfassten Daten. Eine Substitution fehlender Werte wurde nicht vorgenommen. Bei der Auswertung von kategorialen Variablen anhand von absoluten Anzahlen und Prozentangaben wurden fehlende Werte als separate Kategorie („keine Angabe“) betrachtet, sodass sich die Prozentangaben auf $100 \%$ addieren. Patienten, bei denen ein maßgeblicher Wert fehlte, wurden bei bestimmten Auswertungen nicht berücksichtigt. In den Tabellen wurde für jede Auswertung die Anzahl der berücksichtigten („N“) und die der nicht berücksichtigten Patienten („fehlend“) angegeben. Es erfolgte eine multiple logistische Regression der Parameter Alter, Geschlecht und GERD-Q-Score im Hinblick auf die Prädiktion des Therapieerfolgs (primäres Studienziel).

\section{Ergebnisse}

In die Studie wurden insgesamt 155 Patienten aufgenommen. Für 2 Patienten liegt keine Dokumentation einer Alginat-Einnahme vor, sodass die analysierte Studienpopulation 153 Patienten
- Tab. 1 Demografische und klinische Daten der Studienpatienten.

\begin{tabular}{|c|c|c|c|}
\hline \multirow[t]{2}{*}{ Anzahl der Studienpatienten } & \multicolumn{3}{|l|}{153} \\
\hline & \multicolumn{2}{|l|}{ weiblich } & männlich \\
\hline \multirow[t]{2}{*}{ Geschlecht } & $82(53,6 \%)$ & \multicolumn{2}{|c|}{$71(46,4 \%)$} \\
\hline & Median & Q1, Q3 & Range \\
\hline Alter & 57 & 48,67 & $18-85$ \\
\hline Dauer der Reflux-Krankheit (Jahre) & 8,0 & 4,14 & $1-50$ \\
\hline Dauer der PPI-Einnahme (Jahre) & 6,0 & 3,10 & $0-30$ \\
\hline
\end{tabular}

umfasste. Insgesamt 6 Patienten kamen nicht zur Abschlussvisite und bei 1 Patienten fehlt die abschließende Beurteilung der Therapiezufriedenheit. Die demografischen und klinischen Daten der Patienten finden sich in $>$ Tab. 1. Die Hauptsymptomlast mit Einschränkung der Lebensqualität wurde von den Patienten postprandial und in der Nacht angegeben ( $\boldsymbol{A}$ Abb. 1). Dieses Symptommuster korrelierte mit den Einnahmezeitpunkten des Alginats während der Studienphase.

2 Patienten beendeten die Therapie mit Alginat nach 2 bzw. 8 Tagen. Patienten, die die 14-tägige Behandlungsphase beendeten, nahmen im Mittel 38,0 (SD 27,2) Sachets mit einer Spannbreite zwischen 1 und 112 ein. Der durchschnittliche Verbrauch pro Behandlungstag lag bei 3,3 (SD 2,0), eine relevante Variabilität von Tag-zu-Tag war nicht zu erkennen. 107 von 149 Patienten (72\%) mit Abschlussdokumentation berichteten eine Besserung ihrer Therapiezufriedenheit um mindestens 1 Punkt auf der Likert-Skala, 35 fanden ihre Situation unverändert und 7 gaben eine Verschlechterung an ( $\mathbf{A} \mathbf{b} \mathbf{b} . \mathbf{2}$ ). Jüngere Patienten (Alter $\leq 57$ Jahre) und Frauen zeigten einen Trend zu mehr Unzufriedenheit zu Beginn der Therapie. Alter und Geschlecht hatten jedoch keinen Effekt auf die Alginat-Wirksamkeit gemessen an der Therapiezufriedenheit. Dagegen sprachen Patienten mit einem GERDQ-Score von $>8$ besser auf die Therapie an als solche mit einem Score $\leq 8$ (Odds-Ratio 0,421 [0,189; 0,940], p = 0,0348).

Am Ende der Therapie wurden persistierende Reflux-Symptome seltener beklagt (62,3\% vs. $92 \%$ zu Beginn), PPI-Nebenwirkungen wurden etwas seltener angegeben (6,6\% vs. 7,8\%), die Anteile der Patienten mit Bedenken vor einer Langzeittherapie (36,6\% vs. 19,1\%) und mit Ablehnung einer medikamentösen Dauertherapie (32\% vs. 23 \%) waren rückläufig. Der GERDQ-Score besserte sich von im Mittel 10,7 auf 8,7 am Ende der Therapiephase. Dies war bedingt durch positive Effekte bei Sodbrennen, Regurgitation, Übelkeit, Oberbauchschmerz und Schlafstörungen ( $\triangleright$ Abb. 3 ).

Insgesamt beklagten 27 Patienten unerwünschte Wirkungen der Alginat-Therapie. Im Einzelnen waren dies schlechter Geschmack, Übelkeit, Obstipation, Diarrhoe, Oberbauchschmerzen, allergische Reaktion und Schwindel. 9 Patienten beendeten die Therapie vorzeitig wegen Übelkeit $(4 \times)$, Schwindel $(1 \times)$, vermuteter allergischer Reaktion $(3 \times)$ und Oberbauchschmerzen $(1 \times)$. Schwerwiegende unerwünschte Ereignisse traten nicht auf. 

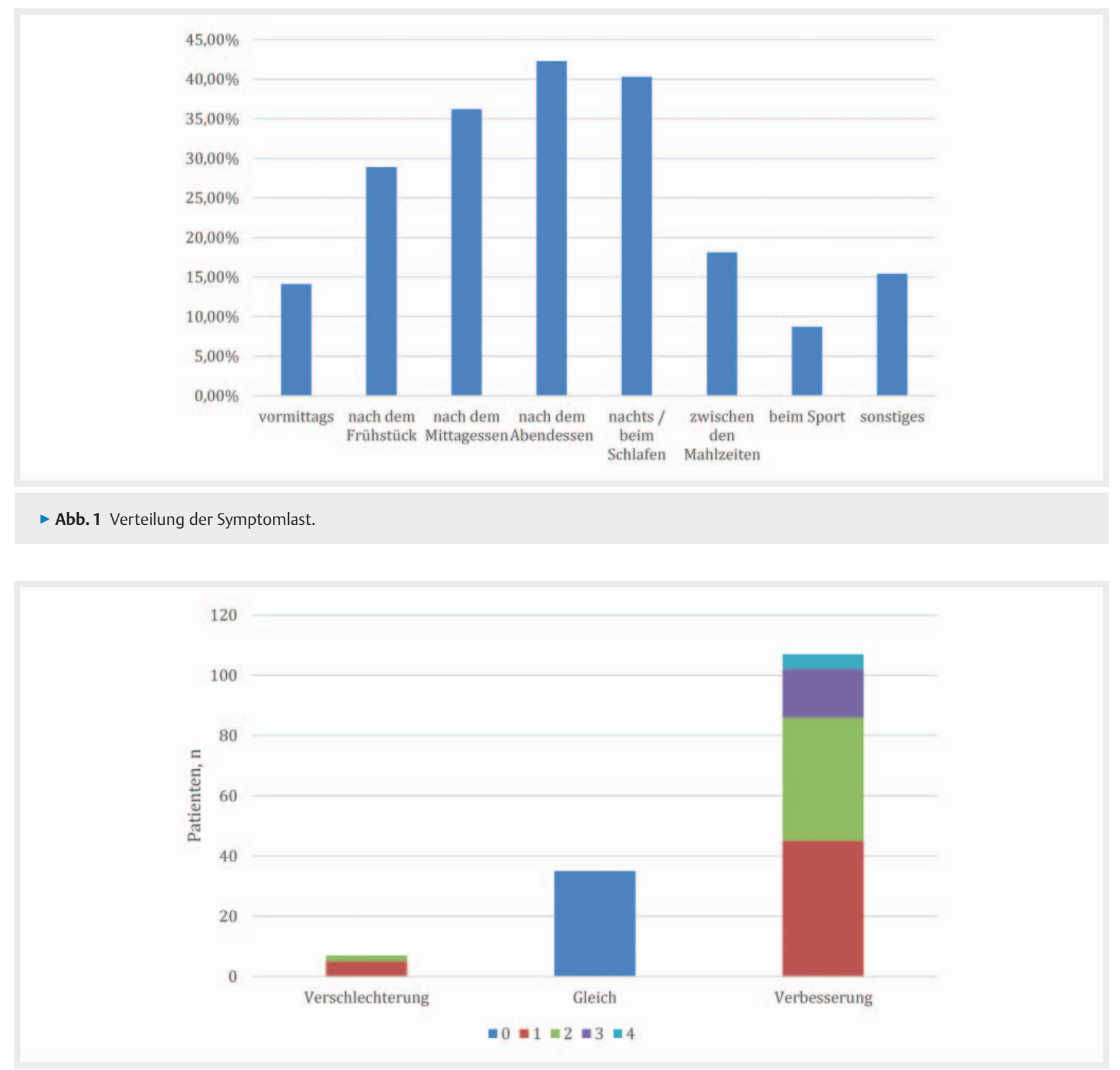

- Abb. 2 Änderung der Patientenzufriedenheit (in Punkten) auf einer 5-stufigen Likert-Skala am Ende der 14-tägigen Therapie mit Alginat als Add-on.

\section{Diskussion}

Zusammenfassend konnten wir in dieser nicht randomisierten, nicht interventionellen und unkontrollierten Studie zeigen, dass die zusätzliche Gabe eines Alginats nach klinischem Bedarf eine neue, einfache und sichere Option ist, die Kontrolle von RefluxSymptomen bei unzureichendem PPI-Effekt und damit die Zufriedenheit mit der Reflux-Therapie zu verbessern.

Grundsätzlich gibt es 3 pathophysiologisch begründete Optionen, die GERD zu behandeln: Säurekontrolle, Reflux-Kontrolle und Beeinflussung der ösophagealen Sensitivität. PPI reduzieren die Azidität des Mageninhalts, beeinflussen den Reflux selbst allerdings kaum. Damit gelingt es, peptische Läsionen des Ösophagus zur Abheilung zu bekommen [11, 12]. Die Entstehung von Symptomen ist komplex und kann sicher nicht allein auf eine Säureexposition der ösophagealen Mukosa reduziert werden [13]. Es ist daher nicht verwunderlich, dass ein substantieller Anteil der Patienten symptomatisch bleibt. In randomisierten und kontrollierten Studien haben 50 \% der Patienten weiterhin Symptome, die von $30 \%$ der Patienten als belästigend empfunden werden [4]. Eine Verbesserung kann sehr wahrscheinlich nicht durch eine Intensivierung der Säurehemmung erzielt werden [14].

Eine medikamentöse Reflux-Kontrolle durch Reduktion transienter Relaxationen des unteren Ösophagussphinkters und eine Erhöhung des Ruhedrucks gelingt mit Baclofen [15]. Hierdurch kann die Wirksamkeit einer PPI-Therapie verbessert werden, so- 


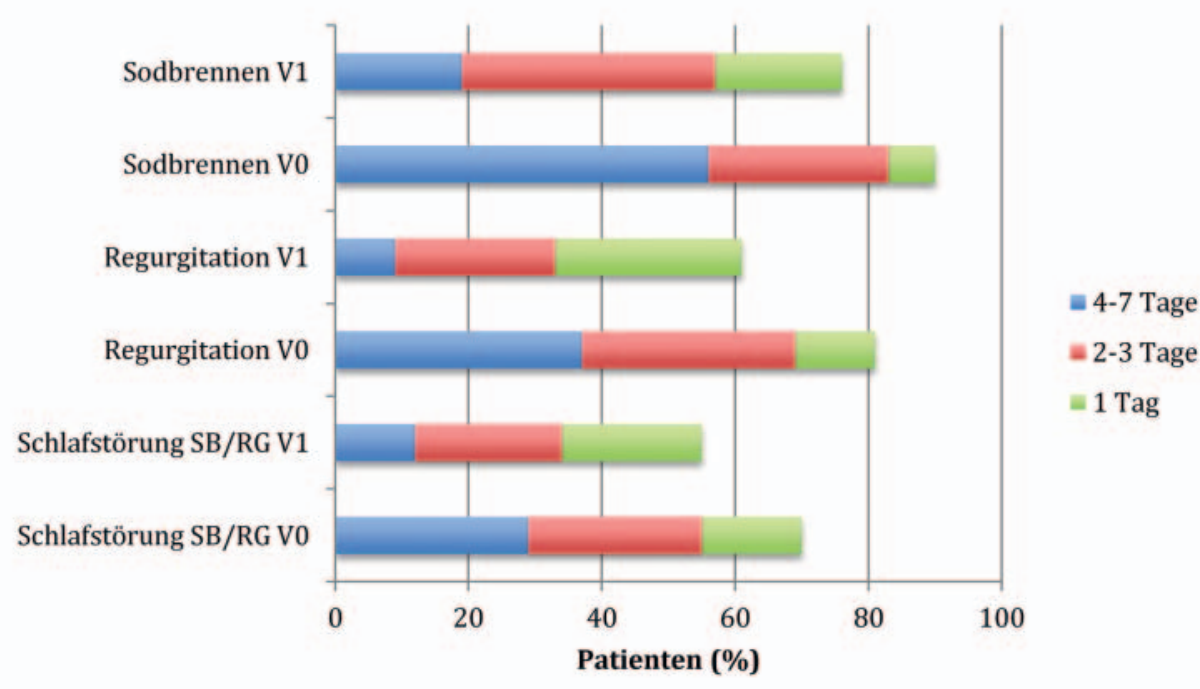

- Abb. 3 Häufigkeit von typischen Reflux-Symptomen und assoziierten Schlafstörungen in der Woche vor der Einschlussvisite (V0) und in der Woche vor der Abschlussvisite (V1) (SB: Sodbrennen, RG: Regurgitation).

dass eine Kombination aus Säure- und Reflux-Kontrolle prinzipiell sinnvoll erscheint [16]. Allerdings ist Baclofen aufgrund seines Nebenwirkungsprofils für eine Dauertherapie eher nicht geeignet, und die Entwicklung von besser verträglichen Reflux-Blockern wurde von der Pharmaindustrie eingestellt.

Eine wesentliche Ursache für insbesondere postprandiale Reflux-Beschwerden ist die sogenannte Acid-Pocket, eine physiologische Säureansammlung unterhalb der Cardia unmittelbar nach Einnahme einer Mahlzeit [6]. Diese Säurebildung lässt sich durch PPI in Standarddosis nicht verhindern. Aus diesem Grund ist die Acid-Pocket ein wesentlicher Grund für persistierende Symptome unter PPI bei GERD. Alginate fallen bei Säurekontakt aus und eliminieren hierdurch die Acid-Pocket [7]. Dies führt in der Konsequenz zu einer Reduktion der Reflux-Ereignisse [8]. Zusätzlich bildet sich ein Film auf dem Ösophagus-Epithel, der auch noch nach 1 Stunde nachweisbar ist [9]. Damit wirken Alginate über Reflux-Kontrolle und evtl. zusätzlich auch noch über eine Beeinflussung der Sensitivität [9, 17]. Das von uns verwendete Alginat ist aus pharmakologischen Gründen mit einem Antazidum kombiniert, was einen zusätzlichen Effekt für die Säureneutralisation haben kann. In einer Metaanalyse kontrollierter Studien sind Alginate den Antazida eindeutig überlegen [18].

In 2 randomisierten, Placebo-kontrollierten Studien konnte gezeigt werden, dass die 4-mal tägliche Einnahme eines Alginats zusätzlich zur unverändert fortgesetzten PPI-Therapie die Symptomkontrolle um $27 \%$ bzw. $39 \%$ verbessert, sodass bei $56 \%$ bzw. $75 \%$ der Patienten ein adäquater Response erzielt wurde [19, 20]. In einer weiteren konfirmatorischen Studie war dieser Effekt allerdings nicht nachweisbar [21]. Die Analyse zeigte, dass in dieser Studie zum einen ein unerwartet hoher Placeboeffekt vorlag und zum anderen viele Patienten, die die Kriterien einer funktionellen Dyspepsie erfüllten, eingeschlossen wurden. Da nicht alle Patienten nach allen Mahlzeiten und in der Nacht Symptome haben und Alginate sofort wirken, haben wir in unserer Studie eine bedarfsadaptierte Medikation gewählt und zunächst in einem nicht Placebo-kontrollierten Design untersucht. Die Responserate lag in dem Bereich der beiden oben erwähnten positiven Studien und ist damit eine neue, vielversprechende Option. Da es sich um eine seit langer Zeit bekannte und für die symptomatische Behandlung von Reflux-Beschwerden zugelassene, frei verkäufliche Therapie handelt, ist eine Placebo-kontrollierte Studie u. E. entbehrlich.

Die Sicherung der Diagnose GERD, definiert als belästigende Symptome bzw. Läsionen als Folge von Reflux von Mageninhalt, ist schwierig [22]. Ein diagnostischer Goldstandard existiert nicht, insbesondere sind auch „typische Symptome“ weder hochsensitiv noch spezifisch [23]. Aus diesem Grund werden auch in unserer Studie - wie praktisch in allen GERD-Studien - Patienten sein, deren Symptome nicht auf Reflux beruhen. In unserer Arbeit sprachen Patienten mit einem GERD-Q-Score über 8, die mit höherer Wahrscheinlichkeit tatsächlich eine GERD haben [10], besser auf die Alginat-Therapie an. Dies unterstützt die Annahme, dass die Wirkung des Präparates auf einer Reflux-Kontrolle beruht, zumal wir keine anderen Prädiktoren, wie z. B. Alter oder Geschlecht, identifizieren konnten.

Die Patienten nahmen das Präparat im Schnitt 3-mal am Tag, v. a. nach den Hauptmahlzeiten und zur Nacht. Damit können die Behandlungskosten gegenüber der 4-mal täglichen Verordnung reduziert werden. Darüber hinaus hat diese Verordnungsform den Vorteil, dass eine Therapie nur bei tatsächlichem Bedarf erfolgt. Dies trägt der klinischen Erfahrung Rechnung, dass viele Reflux-Kranke einen fluktuierenden Symptomverlauf haben. In Placebo-kontrollierten Studien fanden sich bisher keine substanzspezifischen Nebenwirkungen [24]. Auch in unserer Studie, die mehr als 2000 Anwendungstage umfasst, traten keine schwerwiegenden bzw. unerwarteten unerwünschten Ereignisse auf.

Zusammenfassend konnten wir zeigen, dass eine bedarfsadaptierte Therapie mit einem Alginat mit dem Ziel der postprandialen 
und nächtlichen Symptomkontrolle eine wirksame und sichere Option ist für GERD-Patienten, die mit einer alleinigen Säurekontrolle durch PPI persistierende Symptome haben. Dies ist auch ein weiterer Beleg für die sinnvolle Kombination der beiden Therapieprinzipien Säurekontrolle und Reflux-Kontrolle. Momentan wird in Leitlinien noch das Prinzip „Säurekontrolle durch PPI first“ empfohlen [25]. Zukünftige Studien sollten evaluieren, unter welchen klinischen Bedingungen die Reflux-Kontrolle durch Alginate als Erstlinientherapie erwogen werden sollte.

\section{KERNAUSSAGEN}

- Durchbruchsymptome unter PPI sind bei GERD-Patienten häufig und beeinträchtigen die Lebensqualität.

- Eine Reflux-Blockade mit einem Alginat-Add-on ist eine wirksame und sichere Option zur Behandlung typischer Reflux-Symptome und zur Verbesserung der Therapiezufriedenheit von GERD-Patienten mit unzureichendem PPI-Effekt.

- Die zusätzliche Einnahme eines Alginats vor dem Schlafengehen verbessert die Schlafqualität von GERD-Patienten mit PPI-Dauertherapie.

- Ein typisches Reflux-Syndrom (Sodbrennen, Regurgitation) ist ein geeigneter klinischer Prädiktor für den Erfolg der Alginat-Therapie.

\section{Interessenkonflikt}

JL: Beratungs- und Vortragstätigkeit für Reckitt Benckiser GL: Vortragstätigkeit für Reckitt Benckiser MM und DB: keine Interessenkonflikte

\section{Sponsor}

Die Studie wurde aus Drittmitteln des Diakonie-Klinikums, Jung-StillingKrankenhaus Siegen finanziert. Die Firma Reckitt Benckiser stellte dem Klinikum einen Forschung-Grant zur Verfügung.

Literatur

[1] Labenz J, Labenz C. Prävalenz und natürlicher Verlauf der gastroösophagealen Refluxkrankheit. Gastroenterologe 2016; 11: 102-109

[2] Labenz J, Koop H. Gastroösophageale Refluxkrankheit - was tun, wenn PPI nicht ausreichend wirksam, verträglich oder erwünscht sind? Dtsch Med Wochenschr 2017; 142: 356-366

[3] Gyawali CP, Fass R. Management of gastroesophageal reflux disease. Gastroenterology 2018; 154: 302-318

[4] El-Serag H, Becher A, Jones R. Systematic review: persistent reflux symptoms on proton pump inhibitor therapy in primary care and community studies. Aliment Pharmacol Ther 2010; 32: 720-737

[5] Labenz ], Labenz G, Stephan D et al. LOPA-Studiengruppe: Insufficient symptom control under long-term treatment with PPI in GERD - fact or fiction? MMW Fortschr Med 2016; 158 (Suppl. 4): 7-11

[6] Sauter M, Fox M. Die Acid Pocket: ein neues Ziel für die Behandlung der gastroösophagealen Refluxkrankheit. Z Gastroenterol 2018. (im Druck)
[7] Kwiatek A, Pandolfino JE, Kahrilas PJ. An alginate-antacid formulation (Gaviscon Double Action Liquid) can eliminate or displace the postprandial 'acid pocket' in symptomatic GERD patients. Aliment Pharmacol Ther 2011; 34: 59-66

[8] Savarino E, de Bortoli N, Zentelin P et al. Alginate controls heartburn in patients with erosive and nonerosive reflux disease. World J Gastroenterol 2012; 18: $4371-4378$

[9] Woodland P, Batista-Lima F, Lee C et al. Topical protection of human esophageal mucosal integrity. Am J Physiol Gastrointest Liver Physiol 2015; 308: 975 - 980

[10] Jones R, Junghard O, Dent J et al. Development of the GerdQ, a tool for the diagnosis and management of gastro-oesophageal reflux disease in primary care. Aliment Pharmacol Ther 2009; 30: 1030-1038

[11] Khan M, Santana J, Donnellan C. Medical treatments in the short term management of reflux oesophagitis. Cochrane Database Syst Rev 2017; 2: CD003244

[12] Boeckxstaens G, El-Serag HB, Smout AJPM et al. Symptomatic reflux disease: the present, the past and the future. Gut 2014; 63: 1185-1193

[13] Bredenoord AJ. Mechanisms of reflux perception in gastroesophageal reflux disease: a review. Am J Gastroenterol 2012; 107: 8-15

[14] Mizuki A, Tetemichi M, Sakakibara T et al. A multicenter, randomized, open-label trial: efficacy of once-daily versus twice-daily double-dose rabeprazole on refractory gastroesophageal reflux disease-related symptoms and quality of life. Curr Ther Res Clin Exp 2016; 79: 1 -7

[15] Vela MF, Tutuian R, Katz PO. Baclofen decreases acid and non-acid postprandial gastro-oesophageal reflux measured by combined multichannel intraluminal impedance and pH. Aliment Pharmacol Ther 2003; 17 : $243-251$

[16] Kook GH, Sifrim D, Lerut T et al. Effect of the GABAB agonist baclofen in patients with symptoms and duodeno-gastro-oesophageal reflux refractory to proton pump inhibitors. Gut 2003; 52: 1397-1402

[17] Kahrilas PJ, McColl K, Fox M et al. The acid pocket: a target for treatment in reflux disease? Am J Gastroenterol 2013; 108: 1058-1064

[18] Leiman DA, Riff BP, Morgan S et al. Alginate therapy is effective treatment for GERD symptoms: a systematic review and meta-analysis. Dis Esophagus 2017; 30: 1 -9

[19] Manabe N, Haruma K, Ito M. Efficacy of adding sodium alginate to omeprazole in patients with nonerosive reflux disease: a randomized clinical trial. Dis Esophagus 2012; 25: $373-380$

[20] Reimer C, Lodrup AB, Smith G et al. Randomised clinical trial: alginate (Gaviscon Advance) vs. placebo as add-on therapy in reflux patients with inadequate response to once daily proton pump inhibitor. Aliment Pharmacol Ther 2016; 43: 899-909

[21] Coyle C, Crawford G, Wilkinson J et al. Randomised clinical trial: addition of alginate-antacid (Gaviscon Double Action) to proton pump inhibitor therapy in patients with breakthrough symptoms. Aliment Pharmacol Ther 2017; 45: 1524-1533

[22] Gyawali CP, Kahrilas PJ, Savarino E et al. Modern diagnosis of GERD: the Lyon Consensus. Gut 2018. (Epub ahead of print)

[23] Vaezi MF, Pandolfino JE, Vela MF et al. White paper AGA: Optimal strategies to define and diagnose gastroesophageal reflux disease. Clin Gastroenterol Hepatol 2017; 15: $1162-1172$

[24] Sun J, Yang C, Zhao H et al. Randomised clinical trial: the clinical efficacy and safety of an alginate-antacid (Gaviscon Double Action) versus placebo for decreasing upper gastrointestinal symptoms in symptomatic gastroesophageal reflux disease (GERD) in China. Aliment Pharmacol Ther 2015; 42: $845-854$

[25] Koop H, Fuchs KH, Labenz ] et al. S2k-Guideline: Gastroesophageal reflux disease guided by the German Society of Gastroenterology. Z Gastroenterol 2014; 52: $1299-1346$ 